Review

\title{
Disruption of Learning Processes by Chemotherapeutic Agents in Child- hood Survivors of Acute Lymphoblastic Leukemia and Preclinical Models
}

\author{
Emily B. Bisen-Hersh ${ }^{1,2 \bowtie}$, Philip N. Hineline ${ }^{2}$, and Ellen A. Walker ${ }^{1,3}$ \\ 1. Neuroscience Program, Temple University, Philadelphia, PA 19122, USA \\ 2. Department of Psychology, Temple University, Philadelphia, PA 19122, USA \\ 3. Department of Pharmaceutical Sciences, Temple University School of Pharmacy, Philadelphia, PA 19140, USA
}

$\triangle$ Corresponding author: Emily Bisen-Hersh, Department of Pharmaceutical Sciences, Temple University School of Pharmacy, 3307 North Broad Street, Philadelphia, PA 19140, USA. Phone: (215) 707-6291; E-mail: emily.bisen-hersh@temple.edu

(C) Ivyspring International Publisher. This is an open-access article distributed under the terms of the Creative Commons License (http://creativecommons.org/ licenses/by-nc-nd/3.0/). Reproduction is permitted for personal, noncommercial use, provided that the article is in whole, unmodified, and properly cited.

Received: 2011.02.28; Accepted: 2011.05.13; Published: 2011.05.19

\begin{abstract}
Objective: With the survival rate of acute lymphoblastic leukemia (ALL) surpassing 90 percent within this decade, new research is emerging in the field of late effects. A review of the research investigating the relationship of treatment regimens for ALL to specific late effect deficits, underlying mechanisms, and possible remediation is warranted to support continued studies.

Methods: The clinical literature was briefly surveyed to describe the occurrence and topography of late effects, specifically neurocognitive deficits. Additionally, the preclinical literature was reviewed to uncover potential underlying mechanisms of these deficits. The advantages of using rodent models to answer these questions are outlined, as is an assessment of the limited number of rodent models of childhood cancer treatment.

Results: The literature supports that childhood survivors of ALL exhibit academic difficulties and are more likely to be placed in a special education program. Behavioral evidence has highlighted impairments in the areas of attention, working memory, and processing speed, leading to a decrease in full scale IQ. Neurophysiological and preclinical evidence for these deficits has implicated white matter abnormalities and acquired brain damage resulting from specific chemotherapeutic agents commonly used during treatment.

Conclusions: The exact role of chemotherapeutic agents in learning deficits remains mostly unknown. Recommendations for an improved rodent model of learning deficits in childhood cancer survivors are proposed, along with suggestions for future directions in this area of research, in hopes that forthcoming treatment regimens will reduce or eliminate these types of impairments.
\end{abstract}

Key words: childhood cancer, cognitive late effects, acute lymphoblastic leukemia, preclinical models, chemotherapy

\section{Late Effects of Childhood Cancer}

Out of 10 million cancer survivors alive in the United States today, at least 270,000 were diagnosed when they were under the age of 21 [1]. With the advancement of cancer treatment over the past few decades, new interest focuses on "late effects," chronic and progressive conditions associated with successful completion of cancer therapy, which are now prevalent among long-term cancer survivors. Such effects 
usually present three or more years post-diagnosis, with only one in three survivors remaining free of any long-term problems [2,3]. Late effects among childhood cancer survivors are so pervasive that the Children's Oncology Group has issued a recommendation for regular evaluation to monitor development after treatment, a guideline that is often difficult to follow due to time-consuming and expensive assessments [4].

Included among a plethora of chronic medical conditions are cognitive late effects, presumably resulting from chemotherapy administered to the central nervous system (CNS) during a time of rapid brain development [3,5]. For instance, childhood cancer survivors, compared to siblings, are 10 times more likely to have severe cognitive deficits, and are significantly less likely to complete high school or to complete higher education after graduation $[3,6]$. In adulthood, frequency of impairment in areas of task efficiency, memory, and emotional regulation is 50 percent higher among survivors of childhood cancer compared to siblings [7].

Among childhood cancer survivors, acute lymphoblastic leukemia (ALL) is the most common diagnosis [8]. Due to the advancement of treatment regimens, it is now expected that over 90 percent of children diagnosed with ALL will enter into long-term remission, giving it the highest survival rate for pediatric cancers [9]. Extensive reviews of the clinical literature surrounding cognitive late effects associated with ALL treatment exist $[10,11]$, whereas evaluation of cognitive late effects in preclinical models of young rodents is lacking. Therefore, the objective of the current review is to highlight the benefits of using preclinical models to complement clinical research in this area.

\section{Acute Lymphoblastic Leukemia (ALL)}

Leukemias represent about one-third of childhood cancers, with ALL accounting for 75 percent of pediatric leukemia cases, making it the most common form of cancer in children and adolescents [12]. Out of 4,000 cases diagnosed annually in the United States, two-thirds are in these age groups [9]. ALL, a malignant disorder of lymphoid cells, results when a surplus of stem cells develop into lymphocytes, a type of white blood cell also referred to as leukemic cells. These cells are not able to fight infection and leave less room for healthy cells and platelets [9]. Additionally, since these cells are found in bone marrow they are transported by the circulatory system to nearly every organ system, including the CNS. Despite possible environmental, genetic, and viral influences, the exact cause of ALL in most cases remains unknown [13].
While ALL is less prevalent in adults, the mortality rate among this population is much higher than in adolescents and children.

Treatment for the majority of ALL subtypes consists of three phases: induction, intensification (consolidation) therapy, and continuation (maintenance) treatment. Although two-thirds of childhood cases are curable with only 12 months of treatment, the vast majority of patients undergo therapy for two years or more [9]. Across medical institutions, chemotherapeutic agents used vary in type and amount, with the most common being methotrexate (MTX), cytosine arabinoside (cytarabine), anthracyclines (such as doxorubicin), asparaginase, mercaptopurine, vincristine, and corticosteroids, presented alone or in combination [9]. Cranial irradiation, or cranial radiation therapy (CRT), once the most common form of CNS prophylaxis, has largely been replaced by intrathecal (IT) and systemic chemotherapy. This change has been made in an effort to eliminate radiation-specific damage to the CNS [14]. Recent regimens have tested whether CRT can be eliminated completely from standard treatment. To date, this has been successful, although alterations in long-term outcome are just beginning to unfold [15].

\section{Academic and Learning Deficits}

Childhood ALL and its treatments are associated with poor academic outcome, with age at diagnosis being the most important education-related risk factor. One study of infant leukemia, defined as children diagnosed at age 12 months or younger, found that 50 percent exhibited learning deficits more than five years after diagnosis, and the risk increased for each month younger in age at the time of treatment [16]. Survivors of ALL have a greater likelihood of being placed in a special education program, earn lower grades in school on average, and reach a lower educational level than their siblings $[17,18]$. While ALL treatment during childhood is related to overall poor academic performance, clear learning deficits may not arise until four or five years after the initiation of treatment [19]. Interestingly, poor academic performance is not correlated with frequent absenteeism from school, as may seem to be a plausible explanation due to intensive treatments [20].

Given the fact that past treatment for ALL commonly included CRT, the majority of studies on this topic have included radiation as part of participant treatment, but rarely without simultaneous use of chemotherapy [2]. General measures of intellectual functioning were used in these studies, such as the Wechsler Intelligence Scales [21]. Reports of scores declining for at least seven years following treatment 
including cranial irradiation have been noted [22]. It has been suggested that childhood cancer survivors display a decreased rate of learning new information and acquiring new skills, leading to a decline in IQ score $[2,23]$.

Comparisons between CNS prophylaxes involving CRT or IT therapy have yielded mixed results, with several studies finding no significant difference between treatment groups. For example, while a 12-point difference in mean IQ score was found between ALL patients and controls, including sibling and solid tumor control groups, this result was irrespective of CNS prophylaxis type [24]. The observation that the radiation and non-radiation treatment groups both demonstrated deficits may indicate synergistic effects between MTX and cytarabine, with the latter increasing the neurotoxic effects of the former, creating a result similar to CRT. Along these lines, no significant influence of treatment group was found between children who received IT MTX in addition to systemic (IV) MTX, a lower dose of CRT, or a higher dose of CRT, although 22 to 30 percent of children displayed a clinically significant decline in IQ [23]. Other chemotherapeutic agents were used in later phases of treatment, however, which may have played a role in the findings. Alternatively, systemic MTX may potentiate the neurotoxic effects of IT MTX by affecting the brain via indirect pathways [25]. High doses of MTX administered IV may reduce vascularization of the brain, particularly hippocampal blood vessel density [26].

Loss of intellectual functioning, characterized by IQ level, is considered to be secondary, resulting from a range of core deficits. Among these are deficits in attention, working memory, and processing speed [13]. Most studies examining neurocognitive impairments in ALL survivors have focused on deficits in attention, since severe attentional problems occur in approximately one-quarter of survivors, though findings vary depending on the specific subtype of attention examined [27]. Deficits in the targeting, recalling, and manipulating of information to guide goal-directed behavior have been noted up to seven years post-treatment $[28,29]$. In addition, ALL survivors performed poorly on a visual attention task, which required the child to shift attention between the local and global level of stimuli [30]. Children diagnosed younger than 54 months of age exhibit difficulties in both fundamental and complex attention skills. Children diagnosed older than 54 months of age have difficulty with more complex skills only, such as active mental switching and sustained attention [31]. It should be noted, however, that deficits in more complex skills likely arise from reduced fluency in sim- pler, component skills, even if these types of deficits did not reach significance. In addition to age at diagnosis, treatment intensity also may impact attentional functioning, with children given intensified treatments displaying more extensive and widespread difficulties [32].

From a developmental standpoint, disruption in basic skills may not become apparent until difficulties with higher-level abilities surface years later, when emergence of complex repertoires from component skills do not occur in normal progression. One proposed theory of ALL survivors' decrease in IQ level links the cognitive skills of processing speed, working memory, and fluid intelligence, with processing speed playing a significant role in the development of working memory [33]. In turn, working memory underlies the development of higher-level reasoning and fluid intelligence. Evidence for impaired working memory and slowed information processing has been found for ALL survivors given chemotherapy-only treatment, especially when multiple pieces of information are involved [34,35,36,37].

Although the substitution of IT chemotherapy for cranial irradiation has possibly reduced the severity of the impairments outlined above [38,39], evidence of long-term neurocognitive deficits in ALL survivors still exists $[10,11,36]$. One such study examined the learning and academic functioning of children who received chemotherapy alone as treatment for ALL. As compared to newly diagnosed patients, children who had completed a 3-year chemotherapy-only regimen showed greater cognitive impairments and were more likely to have diagnosable learning disabilities as well, despite the fact that no learning difficulties had been identified for these children prior to their ALL diagnosis [19]. In a comparison of IT MTX and triple IT therapy (including MTX, cytarabine, and hydrocortisone), no significant difference in level of cognitive impairment was found between groups, although the MTX group displayed a slightly slower processing speed [40]. Recent studies suggest that up to 40 percent of childhood cancer survivors given chemotherapy-only treatment may experience neurocognitive deficits years later [4].

Much of the clinical findings from longitudinal studies on the impact of chemotherapy-only treatment for ALL have been inconsistent, possibly because of differences among methodological approaches and medical protocols. There are multiple methodological challenges in longitudinal cognitive assessment, such as selection of appropriate neurocognitive domains and control groups, differences in criteria for impairment, and repeated testing [41]. It is suggested that the percentage of ALL survivors 
experiencing neurocognitive deficits may rise to 70 percent depending on the specific type of cognitive domain assessed, particularly in the area of working memory [42]. In addition, ALL treatment protocols often differ in a multitude of ways, leaving the question of which agents and doses affect neurocognitive outcome largely unknown [11,12]. Given that treatment is comprised of multiple phases, each with a unique combination of drugs, it has been demonstrated that even a single agent substitution within the complex protocol alters whether or not cognitive late effects appear [43].

\section{Neurophysiological Evidence}

Neurophysiological evidence for deficits common among childhood ALL survivors have implicated white matter abnormalities, which may result from a disruption of the myelinization process occurring during childhood [2]. Along with white matter hypodensity, MTX also causes leukoencephalopathy, multiple necrotic lesions in the periventricular white matter $[13,44]$. Behavioral symptoms correlated with this type of injury occur gradually over time, beginning with decreased attentiveness and leading to intellectual decline [45]. Impairment in attentional abilities accounted for a significant amount of the variance relating to reduced volumes of normal-appearing white matter and IQ [46]. Moreover, cumulative (12 to 30) IT doses of MTX correlates positively with deficits observed in neuropsychological tests of IQ, attention, and concentration [47]. The identification of folate pathway genetic polymorphisms that predict childhood cancer patients at-risk for developing attentional impairment following MTX treatment is underway [27].

In general, myelinization appears to follow functional maturation across brain regions [48]. During childhood, one area of the brain undergoing a significant amount of myelinization is the frontal lobe [2]. Since myelinization in this area typically occurs later in development, and the mature frontal lobe characteristically has a high volume of white matter, it may be more vulnerable to damage early in life. Volumetric reductions of the dorsolateral prefrontal cortices, along with the mammillary bodies and caudate nuclei, were examined in survivors three years post-treatment who had received IT chemotherapy [49]. Despite no significant difference in global volumetric brain size, a reduction was found in both the mammillary bodies and dorsolateral prefrontal cortices. The caudate nuclei, thought to develop earlier, were not significantly different in structure from controls. This pattern of abnormality corresponds to noted deficits of memory, processing speed, distracti- bility, and attention in childhood ALL survivors. Advanced neuroimaging techniques that allow for more precise measurement of myelin integrity and degradation have been proposed for use in this population, which include diffusion tensor imaging, quantitative magnetization transfer imaging, and quantitative multiple exponential T2 measurements [42].

When studied in vitro, primary, non-cancer cells were more vulnerable to the toxic effects of cytarabine, BCNU, and cisplatin than cancer cells. At a dose equivalent to a low-dose cancer treatment regimen, 60 percent of oligodendrocytes were killed within 24 hours. Similarly, at a dose equivalent to the lower end of a high-dose cancer treatment regimen, nearly all oligodendrocytes were killed along with 50 percent of glial-restricted precursor cells [50]. Recent evidence supports that chemotherapeutic agents once thought unable to readily cross the blood-brain barrier, including doxorubicin, reduce neural cell proliferation in the dentate gyrus [51]. This mechanism has not yet been evaluated from a developmental standpoint, which is important to consider since the blood-brain barrier of a child is still undergoing development and is therefore more susceptible to chemotherapy-induced CNS damage.

A multitude of potential mechanisms underlying chemotherapy-induced cognitive deficits have been proposed, although not specifically in relation to childhood cancer survivors. In addition to white matter damage and reduced cell proliferation discussed above, proposed additional mechanisms include increased oxidative stress, neuroinflammation, reduced blood flow, deregulation of the immune response, and deficits in DNA-repair mechanisms [52,53 for extensive reviews]. Examining the deleterious effects of chemotherapeutic agents and correlating these effects with behavioral measures using neural cells, immature brains, and mature brains may provide valuable insight into the mechanisms underlying cognitive late effects in ALL survivors.

\section{The Benefits of Preclinical Rodent Models}

It is not feasible in a human patient population to conduct the type of empirically valid research study that is needed to answer the interrelated questions of which drugs, combinations, or doses are most at risk. Besides the ethical implications of tampering with treatment regimens that have proven successful in curing ALL, assessment of improved cognitive outcome would take years [54]. Additionally, it is difficult to separate drug effects from other factors, such as physiological consequences of cancer or patient depression [55]. Another option, that of retrospective 
studies, does not take into consideration the most recent treatment protocols currently in use. Furthermore, this type of evaluation does not capture the developmental trajectory of the drug effects in question, a particularly important factor related to childhood cancer survivors.

An alternative method to study disruption of learning processes by chemotherapeutic agents is through the use of preclinical models. This type of paradigm allows drug effects to be addressed independently of other possible contributing factors, and provides a rapid way to evaluate many drugs, both in terms of type and dosage. With an average lifespan of about two years, rodents allow effects to be studied within a developmental framework as well. Although many past studies involving chemotherapeutic agents and rodent models have involved drug administration of a single agent $[56,57,58]$, rodent models make it possible to study drug combinations, thus providing a more accurate model of current protocols for childhood cancer treatment. Underlying mechanisms of chemotherapy-induced neurotoxicity such as decreased cell proliferation can only be studied using preclinical models. Drugs that may prevent or alleviate deleterious effects resulting from chemotherapeutic treatment also can be evaluated. For example, there is an emerging literature examining methylphenidate as a possible treatment for cognitive late effects in the childhood cancer survivor population $[59,60,61]$. In addition, sex differences among drug effects can be examined in rodents, for there is some evidence to suggest that girls are more susceptible to the neurotoxic effects of these agents [62].

While studies examining the mechanisms for chemotherapy-induced neurotoxicity in clinical studies are limited, preclinical models in adult rodents have demonstrated that MTX and cytarabine manage to cross the blood-brain barrier following various routes of administration. For instance, cell death and disruption of cell division occurs in vivo following three systemic injections of cytarabine in mice [50]. A single IV dose of MTX (37.5-300 $\mathrm{mg} / \mathrm{kg})$ dose-dependently reduces hippocampal cell proliferation in rats. As the hippocampus is important for learning and memory, detrimental effects on cognitive performance are likely to result if neurogenesis is disrupted. This theory was supported by impairment following MTX treatment on the Morris water maze and novel object recognition tasks, designed to measure spatial and working memory, respectively [63]. Furthermore, intracerebroventricular injections of MTX administered for three alternative days to rats resulted in lowered concentrations of hippocampal brain amines. These findings correlated with results from a conditioned avoidance task, in which adult rats treated with MTX failed to learn to avoid an aversive stimulus relative to controls [64].

Rodent studies have also examined the impact of MTX on the younger, developing brain. For example, MTX $(0.05 \mathrm{mg} / \mathrm{kg}$ or $0.1 \mathrm{mg} / \mathrm{kg}$, i.p.) administered during a critical period of brain development in rat pups leads to a reduced density of synapses in the CA3 field of the hippocampus [65]. Additionally, a model of MTX encephalopathy in young rats was developed by administering intraventricular injections of MTX ( 1 or $2 \mathrm{mg} / \mathrm{kg}$ ). Five repeated doses produced neuropathological changes similar to the damage seen in human patients [66]. However, no behavioral measures of learning were included in these studies.

Preclinical models have been useful to investigate chemotherapy-induced deficits in learning and memory using adult models of breast cancer treatment within a variety of behavioral assays $[67,68,69,70]$. These studies have important implications for other treatment protocols composed of multiple drug agents, such as ALL. For instance, the use of rodent models has highlighted the possibility of drug synergisms, i.e., drug combinations that have a greater effect than either drug alone. While some regimens include drugs known to have specific synergistic effects that aid in treating the cancer itself, unwanted side effects, including cognitive deficits, may result as well [67]. Strategies for attenuating chemotherapy-induced cognitive deficits have been examined in rodent models, including the use of anti-depressants such as fluoxetine to block decreased cell proliferation following treatment with 5-fluorouracil [71]. Fluoxetine also reverses the suppression of neurogenesis produced by MTX in adult rodents [72]. Findings from these types of studies will help physicians make informed choices about treatment options that reduce or eliminate chemotherapy-induced cognitive deficits, as well as ways in which to treat these impairments should they arise [69].

\section{Rodent Models of Childhood Cancer Treat- ment}

Studies using rodent models to elucidate the effects of childhood cancer treatment in young pups have been much more limited. It is exceedingly important to study treatment in developing rodent brains, since neonatal damage has been found to have more severe long-term effects than identical damage in the mature adult rodent brain [73]. Although studies addressing the influence of MTX on the developing rodent brain exist, few include behavioral measures. Additionally, 
previous models of childhood treatment have generally focused on an acute administration of a single chemotherapeutic agent, typically MTX. Of the studies that do include behavioral measures of young pups, many entail assays that seem loosely tied and functionally disconnected from the actual deficits displayed in human childhood cancer survivors.

For instance, the first assessment of cancer treatment on developing brains used young rat pups at PND 17, an age selected because of developmental similarities to human infants. Treatment included CRT alone (1,000 R), MTX alone (5 mg/ kg, i.p.), or a combination of CRT and MTX. Testing with a simultaneous discrimination task began when the rats were 12-14 weeks old. Findings demonstrated that only animals receiving a combination treatment were significantly slower to reach criterion, compared to the rest of the groups [57].

However, in contrast to ALL treatment protocols that include multiple phases of therapy, typically over the course of two years, this study tested a single dose of radiation and/or MTX. According to the authors, this was done because the CNS of rats this age develops at an exceedingly fast rate. This limitation is shared among the majority of studies using rodent models to investigate childhood cancer treatment in young pups. In another study, rat pups 16-17 days old were treated with a single dose of MTX (0.005 $\mathrm{mg} / \mathrm{kg}$, i.p.). At 12-14 weeks of age, animals were tested on conditioned emotional response and conditioned taste aversion tasks. Animals treated with MTX acquired the delay conditioning at a slower rate. Rats also were tested on a conditioned taste aversion task, in which a previously highly palatable fluid was paired with illness. MTX-treated animals failed to display a taste aversion following the first trial, compared to controls, but were equal to controls by the second trial. Given the above results, it was concluded that neonatal rats administered MTX were slower to learn about environmental events [58]. In contrast, no impairments were found in 17-day-old rat pups treated with MTX $(0.005 \mathrm{mg} / \mathrm{kg}$, i.p. $)$ in a similar conditioned taste aversion task, although a different strain of rats was studied. Likewise, no impairment was found on a more complex Pavlovian conditioning task focusing on negative discrimination [56].

As already addressed, given the fact that childhood cancer regimens consist of an array of drugs, it is essential to examine possible interactions that may exist among them. This issue was tackled by studying rats in nine different treatment combinations of MTX (2 or $4 \mathrm{mg} / \mathrm{kg}$, i.p.), prednisolone (18 or $36 \mathrm{mg} / \mathrm{kg}$, i.p.), and CRT (1000 cGy), treated at PND 17-18 [74]. Steroids such as prednisolone are commonly included among the drugs used in ALL treatment, particularly in double and triple IT therapy, and there is evidence to suggest that glucocorticoid steroids potentiate hippocampal damage caused by neurotoxins [75]. In this study, spontaneous behavior was measured for behavioral initiations, as well as time distribution and the sequence of behavioral acts. Greater behavioral deficits were found as the treatment group became more complex. Effects also were dose and sex-dependent, with females displaying altered behavior at lower doses than males [74]. This is consistent with clinical literature suggesting girls may be more susceptible to the aversive effects of these drugs [62]. While prednisolone antagonized MTX, thereby preventing behavioral alterations, at low doses, prednisolone enhanced MTX and CRT-related deficits at high doses [74].

In addition to MTX, cytarabine, vincristine, doxorubicin, and L-asparaginase are commonly mentioned in clinical studies as being part of ALL chemotherapeutic treatment, but information pertaining to the individual contributions of these agents to neurocognitive deficits is limited. These types of evaluations are difficult to assess through clinical studies given that chemotherapeutic agents are often combined. Therefore, the potential involvement of cytarabine, vincristine, doxorubicin, and L-asparaginase cannot be dismissed for their contributions to neurocognitive deficits. Preclinical models have provided evidence of cognitive disruption following administration of these drugs, though research remains limited to adult rodents. To date, cytarabine produced impairment in long-term spatial memory in rats 30 days post-training, but not 1 day post-training, on the Morris water maze [76]. Preclinical research involving vincristine has largely been discussed in relation to disrupted sensory processing, including neuropathies and mechanical sensitivity [77], but impairment in spatial learning as measured by the Morris water maze has been noted at high doses [78]. When administered alone, doxorubicin treatment led to impairment in inhibitory avoidance conditioning in rats, but not on a passive avoidance task in mice $[79,80]$. In combination with cyclophosphamide, doxorubicin produced impairment in contextual fear conditioning and passive avoidance learning in rats $[81,82]$. The link between L-asparaginase and cognition has not yet been examined, but is an important factor to consider for future research in this area. The individual effects of specific corticosteroids, commonly used in combination with chemotherapeutic agents, are correlated with poor cognitive outcome as well. For example, children treated with dexamethasone may be at greater risk for neurocognitive late 
effects, compared to children treated with prednisone [43].

\section{Future Directions}

Limitations of preclinical models of childhood cancer treatment are obvious as several factors complicate this type of research. For instance, the early stages of rodent life are not yet definitively mapped out, although there are some guidelines about developmental milestones such as reflexes and locomotor behavior [83], and age equivalences for the adolescence period in rodents have been proposed [84]. Development of the blood-brain barrier and lack of certain enzymes at early stages will impact how chemotherapeutic agents are metabolized by the body, as well as how the brain is affected. Decisions about dosage and route of administration also need to take these developmental aspects into account and limited pharmacokinetic and pharmacodynamic models are available. Additionally, the short lifespan of rodents means that progression through stages occurs at an accelerated rate. This fact needs to be taken into consideration when choosing behavioral assays, as well as when selecting the points along the lifespan that rodents are to be tested. Nevertheless, use of adult rodents to study the effects of childhood cancer does not take into consideration treatment impact on a developing brain, as opposed to one that is already matured, and is therefore a less valid model.

Translational research entails basic science and clinical practice working together, with each informing the other. Building a bridge between clinical and preclinical research would greatly improve the study of chemotherapy-induced cognitive effects of childhood cancer treatment. While this philosophy has been applied to models of adult cancer, developmentally focused accounts of childhood cancer treatment are limited. Future preclinical research in this area should aim to provide a more accurate model of clinical treatment through alterations in drug selection, treatment regimen, and behavioral measures. Since MTX and cytarabine are commonly administered together during CNS prophylaxis in ALL treatment, it would be valuable to investigate the effects of this specific combination of chemotherapeutic agents. Preclinical models are ideal for parsing apart the individual and combined effects on learning and memory of lesser-studied chemotherapeutic agents used in ALL treatment, such as vincristine, doxorubicin, and asparaginase. Repeated administration of these agents can be examined by treating pre-weanling pups on multiple consecutive days early in development, rather than a single administration of treatment. Rodents can be assessed during various stages of development, including adolescence (PND 35) and adulthood (PND 60), to examine long-term effects of early neurotoxicity.

In order to advance this area of research, appropriate behavioral assays need to be selected that characterize the neurocognitive deficits experienced by ALL survivors. Since the exact mechanisms of chemotherapy-induced cognitive impairment are not yet fully understood, a battery of preclinical assays of learning and memory should be studied, as sensitivity to chemotherapy-induced cognitive deficits differs among tasks in models of adult chemotherapeutic treatment [53]. Paradigms used in rodent models of childhood cancer treatment are much more limited and have typically focused on respondent conditioning. Evaluating effects on instrumental conditioning will provide new insight into the type of learning processes impaired by chemotherapeutic treatment. Impairment on an autoshaping-operant procedure has been demonstrated following treatment with MTX and 5-fluorouracil in adult mice [67], whereas no impairment was found on the five choice serial reaction time task following treatment with paclitaxel [85]. While both of these tasks measure instrumental conditioning, the two procedures differ in terms of length of training. This distinction would be interesting to examine within a developmental context, both in regard to learning deficit as well as potential rehabilitation. Other cognitive assessments that have demonstrated impairments following adult chemotherapeutic treatment are useful for a developmentally-focused model of ALL, such as novel object recognition and the Morris water maze, which measure working and spatial memory, respectively $[76,86]$. It is particularly important to include tasks of sustained attention or vigilance, as these deficits are pervasive among childhood cancer survivors. In addition to the five choice serial reaction time task previously discussed, the Go/No-go task also measures sustained attention through reinforcer delivery being contingent upon responding in the presence of a cue [87].

The preclinical research discussed here all involved healthy rodents. The validity of using healthy animals to study chemotherapy-induced neurotoxicity is supported by the finding that tumor presence does not potentiate a MTX-induced decrease of cell proliferation in the hippocampus [88]. A logical next step will be to investigate learning deficits using a model bearing an ALL-related cancer. This will enable insight into the interaction between chemotherapy and the ALL cancer itself. However, using immunosuppressed rodents may create a new set of challenges for conducting behavioral testing, particularly if 
equipment is shared with rodents that do not have a compromised immune system.

\section{Conclusions}

In summary, with survival rate for childhood cancers on the rise, it is imperative that new research focuses on what happens after a child enters into remission. As has been recommended by the Children's Oncology Group, it is essential that children and their parents be informed of potential late effects [1]. Survivors need to be closely monitored by healthcare workers and school personnel for emerging medical complications and/or academic difficulties, which, as noted in the current review, may not arise until several years after treatment has ended. Further guidelines relating to childhood cancer survivors are outlined in the Long-Term Follow-Up Program Resource Guide, available online from the Children's Oncology Group [1]. Attempts to create reliable and valid assessment tools that can quickly identify at-risk survivors are currently underway [89]. Additionally, assessment of current multiple-phase treatment protocols is needed to evaluate specific drug effects that may contribute to cognitive late effects. These findings will help physicians make informed choices about treatment options that reduce or eliminate chemotherapy-induced neurocognitive deficits, including attention and working memory, the presence of which have been supported by both behavioral and neurophysiological evidence. This effort will be greatly facilitated through converging lines of research from both the clinical and preclinical literatures. Currently, a child's fight with cancer does not end with remission, but hopefully, with new efforts focusing on research in the area of late effects, one day it will.

\section{Acknowledgements}

A portion of this review was completed in fulfillment of the requirements for the doctoral degree of Emily Bisen-Hersh. This work was supported by NIH (R01 CA129092 to EAW).

\section{Conflict of Interest}

The authors have declared that no conflict of interest exists.

\section{References}

1. [Internet] Children's Oncology Group. Establishing and enhancing services for childhood cancer survivors: long-term follow-up program resource guide. http://www.survivorshipguidelines.org/

2. Moleski M. Neuropsychological, neuroanatomical, and neurophysiological consequences of CNS chemotherapy for acute lymphoblastic leukemia. Arch Clin Neuropsych. 2000; 15: 603-630.

3. Oeffinger $\mathrm{KC}$, Mertens AC, Sklar CA, et al. Chronic health conditions in adult survivors of childhood cancer. N Engl J Med. 2006; 355: 1572-1582.

4. Krull KR, Okcu MF, Potter B, et al. Screening for neurocognitive impairment in pediatric cancer long-term survivors. J Clin Oncol. 2008; 26: 4138-4143.

5. Bowers DC, Liu Y, Leisenring W, et al. Late-occurring stroke among long-term survivors of childhood leukemia and brain tumors: a report from the childhood cancer survivor study. J Clin Oncol. 2006; 24: 5277-5282.

6. Mulrooney DA, Dover DC, Li S, et al. Twenty years of follow-up among survivors of childhood and young adult acute myeloid leukemia. Cancer. 2008; 112: 2071-2079.

7. Kadan-Lottick NS, Zeltzer LK, Liu Q, et al. Neurocognitive functioning in adult survivors of childhood non-central nervous system cancers. JNCI. 2010; 102: 881-893.

8. Leisenring WM, Mertens AC, Armstrong GT, et al. Pediatric cancer survivorship research: experience of the childhood cancer survivor study. J Clin Oncol. 2009; 27: 2319-2327.

9. Pui C \& Evans WE. Treatment of acute lymphoblastic leukemia. N Engl J Med. 2006; 354: 166-178.

10. Buizer AI, de Sonneville LMJ, \& Veerman AJP. Effects of chemotherapy on neurocognitive function in children with acute lymphoblastic leukemia: a critical review of the literature. Pediatr Blood and Cancer. 2009; 52: 447-454.

11. Janzen LA, \& Spiegler BJ. Neurodevelopmental sequelae of pediatric acute lymphoblastic leukemia and its treatment. Dev Dis Rev. 2008; 14: 185-95.

12. Butler RW \& Haser JK. Neurocognitive effects of treatment for childhood cancer. Ment Retard Dev Dis Rev. 2006; 12: 184-191.

13. Mulhern RK \& Butler RW. Neurocognitive sequelae of childhood cancers and their treatment. Pediatr Rehabilitation. 2004; 7: 1-14.

14. Stehbens JA, Kaleita TA, Noll RB, et al. CNS prophylaxis of childhood leukemia: what are the long-term neurological, neuropsychological, and behavioral effects? Neuropsychol Rev. 1991; 2: 147-177.

15. Pui C, Campana D, Pei D, et al. Treatment of childhood acute lymphoblastic leukemia without prophylactic cranial irradiation. N Engl J Med. 2009; 360: 2730-2741.

16. Leung $W$, Hudson $M, Z$ Zhu $Y$, et al. Late effects of survivors of infant leukemia. Leukemia. 2000; 14: 1185-1190.

17. Haupt R, Fears TR, Robinson LL, et al. Educational attainment in long-term survivors of childhood acute lymphoblastic leukemia. JAMA. 1994; 272: 1427-1432.

18. Kingma A, Rammeloo LAJ, van der Does-van den Berg A, et al. Academic career after treatment for acute lymphoblastic leukaemia. Arch Dis Child. 2000; 82: 353-357.

19. Brown RT, Madan-Swain A, Pais R, et al. Chemotherapy for acute lymphocytic leukemia: cognitive and academic sequelae. J Pediatr. 1992; 121: 885-889.

20. Taylor, HG, Albo VC, Phebus C, et al. Post irradiation treatment outcomes for children with acute lymphocytic leukemia. J Pediatr Psychol. 1987; 12: 394-411.

21. Moss HA, Nannis ED, \& Poplack DG. The effects of prophylactic treatment of the central nervous system on the intellectual functioning of children with acute lymphocytic leukemia. Am J Med. 1981; 71: 47-52.

22. Jankovic M, Brouwers P, Valsecchi MG, et al. Association of 1800 cGy cranial irradiation with intellectual function in children with acute lymphoblastic leukaemia. Lancet. 1994; 344: 224-227.

23. Mulhern RK, Fairclough D, \& Ochs J. A prospective comparison of neuropsychologic performance of children surviving leuke- 
mia who received 18-Gy, 24-Gy, or no cranial irradiation. J Clin Oncol. 1991; 9: 1348-1356.

24. Giralt J, Ortega JJ, Olive T, et al. Long-term neuropsychologic sequelae of childhood leukemia: comparison of two CNS prophylactic regimens. Int J Radiat Oncol. 1992; 24: 49-53.

25. Espy KA, Moore IM, Kaufmann PM, et al. Chemotherapeutic CNS prophylaxis and neuropsychologic change in children with acute lymphoblastic leukemia: a prospective study. J Pediatr Psychol. 2001; 26: 1-9.

26. Seigers R, Timmermans J, van der Horn HJ, et al. Methotrexate reduces hippocampal blood vessel density and activates microglia in rats but does not elevate central cytokine release. Behav Brain Res. 2010; 207: 265-272.

27. Krull KR, Brouwers P, Neelam J, et al. Pathway genetic polymorphisms are related to attention disorders in childhood leukemia survivors. J Pediatr. 2008; 152: 101-105.

28. Rodgers J, Horrocks J, Britton PG, et al. Attentional ability among survivors of leukaemia. Arch Dis Child. 1999; 80: 318-323.

29. Langer T, Martus P, Ottensmeier H, et al. CNS late-effects after ALL therapy in childhood. Part III: Neuropsychological performance in long-term survivors of childhood ALL: impairments of concentration, attention, and memory. Med Pediatr Oncol. 2002; 38: 320-328.

30. Schatz J, Kramer JH, Ablin AR, et al. Visual attention in long-term survivors of leukemia receiving cranial radiation therapy. J Int Neuropsychol Soc. 2004; 10: 211-220.

31. Lockwood KA, Bell TS, \& Colegrove RW. Long-term effects of cranial radiation therapy on attentional functioning in survivors of childhood leukemia. J Pediatr Psychol. 1999; 24: 55-66.

32. Buizer AI, de Sonneville LMJ, van den Heuvel-Eibrink MM, et al. Chemotherapy and attentional dysfunction in survivors of childhood acute lymphoblastic leukemia. Pediatr Blood Cancer. 2005; 45: 281-290.

33. Schatz J, Kramer JH, Ablin A, et al. Processing speed, working memory, and IQ: a developmental model of cognitive deficits following cranial radiation therapy. Neuropsychology. 2000; 14 : 189-200.

34. Ashford J, Schoffstall C, Reddick WE, et al. Attention and working memory abilities in children treated for acute lymphoblastic leukemia. Cancer. 2010; 116: 4638-4645.

35. Mennes M, Stiers P, Vandenbussche E, et al. Attention and information processing in survivors of childhood acute lymphoblastic leukemia treated with chemotherapy only. Pediatr Blood Cancer. 2005; 44: 479-486.

36. Campbell LK, Scaduto M, Sharp W, et al. A meta-analysis of the neurocognitve sequelae of treatment for childhood acute lymphocytic leukemia. Pediatr Blood Cancer. 2007; 49: 65-73.

37. Jain N, Brouwers P, Okcu M, et al. Sex-specific attention problems in long-term survivors of pediatric acute lymphoblastic leukemia. Cancer. 2009; 115: 4238-4245.

38. Spiegler BJ, Kennedy K, Maze R, et al. Comparison of long-term neurocognitive outcomes in young children with acute lymphoblastic leukemia treated with cranial radiation or high-dose or very high-dose intravenous methotrexate. J Clin Oncol. 2006; 24: 3858-3864.

39. Waber DP, Turek J, Catania L, et al. Neuropsychological outcomes from a randomized trial of triple intrathecal chemotherapy compared with 18 gy cranial radiation as CNS treatment in acute lymphoblastic leukemia: findings from Dana-Farber cancer institute ALL consortium protocol 95-01. J Clin Oncol. 2007; 25: 4914-4921.

40. Kadan-Lottick NS, Brouwers P, Breiger D, et al. Comparison of neurocognitive functioning in children previously randomly assigned to intrathecal therapy for the treatment of childhood acute lymphoblastic leukemia. J Clin Oncol. 2009; 27: 5986-5992.
41. Vardy J, Wefel JS, Ahles T, et al. Cancer and cancer-therapy related cognitive dysfunction: an international perspective from the venice cognitive workshop. Ann Oncol. 2008; 19: 623-629.

42. Reddick WE, \& Conklin HM. Impact of acute lymphoblastic leukemia therapy on attention and working memory in children. Expert Rev Hematol. 2010; 3: 655-659.

43. Waber DP, Carpentieri SC, Klar N, et al. Cognitive sequelae in children treated for acute lymphoblastic leukemia with dexamethasone or prednisone. J Pediatr Hematol Oncol. 2000; 22: 206-213.

44. Ochs, JJ. Neurotoxicity due to central nervous system therapy for childhood leukemia. Am J Pediatr Hematol Oncol. 1989; 11: 93-105.

45. Lee YY, Nauert C, \& Glass P. Treatment-related white matter changes in cancer patients. Cancer. 1986; 57: 1473-1482.

46. Reddick WE, White HA, Glass JO, et al. Developmental model relating white matter volume to neurocognitive deficits in pediatric brain tumor survivors. Cancer. 2003; 97: 2512-2519.

47. Steinberg S, Hartmann R, Wisniewski S, et al. Late sequelae of CNS recurrence of acute lymphoblastic leukemia in childhood. Klin Padiatr. 1998; 210: 200-206.

48. Filley CM. The behavioral neurology of cerebral white matter. Neurology. 1998; 50: 1535-1540.

49. Ciesielski KT, Lesnik PG, Sanders JA, et al. MRI morphometry of mamillary bodies, caudate nuclei, and prefrontal cortices after chemotherapy for childhood leukemia: multivariate models of early and late developing memory subsystems. Behav Neurosci. 1999; 113: 439-450.

50. Dietrich J, Han R, Yang Y, et al. CNS progenitor cells and oligodendrocytes are targets of chemotherapeutic agents in vitro and in vivo. J Biol. 2006; 5: 22.1-22.

51. Janelsins MC, Roscoe JA, Berg MJ, et al. IGF-1 partially restores chemotherapy-induced reductions in neural cell proliferation in adult C57BL/ 6 mice. Cancer Invest. 2010; 28: 544-553.

52. Ahles TA \& Saykin AJ. Candidate mechanisms for chemotherapy-induced cognitive changes. Nat Rev Cancer. 2007; 7: 192-201.

53. Seigers R \& Fardell JE. Neurobiological basis of chemotherapy-induced cognitive impairment: a review of rodent research. Neurosci Biobehav Rev. 2011; 35: 729-741.

54. Rubenstein CL, Varni JW, \& Katz ER. Cognitive functioning in long-term survivors of childhood leukemia: a prospective analysis. J Dev Behav Pediatr. 1990; 11: 301-305.

55. Raffa RB, Duong PV, Finney J, et al. Is 'chemo-fog' /'chemo-brain' caused by cancer chemotherapy? J Clin Pharm Ther. 2006; 31: 129-138.

56. Stock HS, Rosellini RA, Abrahamsen GC, et al. Methotrexate does not interfere with an appetitive pavlovian conditioning task in sprague-dawley rats. Physiol Behav. 1995; 58: 969-973.

57. Yadin E, Bruno L, Micalizzi M, et al. An animal model to detect learning deficits following treatment of the immature brain. Child's Brain. 1983; 10: 273-280.

58. Yanovski JA, Packer RJ, Levine JD, et al. An animal model to detect the neuropsychological toxicity of anticancer agents. Med Pediatr Oncol. 1989; 17: 216-221.

59. Conklin HM, Helton S, Ashford J, et al. Predicting methylphenidate response in long-term survivors of childhood cancer: a randomized, double-blind, placebo-controlled, crossover trial. J Pediatr Psychol. 2010; 35: 144-155.

60. Conklin HM, Khan RB, Reddick WE, et al. Acute neurocognitive response to methylphenidate among survivors of childhood cancer: a randomized, double-blind, cross-over trial. J Pediatr Psychol. 2007; 32: 1127-1139.

61. Waber DP, Tarbell NJ, Fairclough D, et al. Cognitive sequelae of treatment in childhood acute lymphoblastic leukemia: cranial radiation requires an accomplice. J Clin Oncol. 1995; 13: 2490-2496. 
62. Waber DP, Tarbell NJ, Kahn CM, et al. The relationship of sex and treatment modality to neuropsychologic outcome in childhood acute lymphoblastic leukemia. J Clin Oncol. 1992; 10: 810-817.

63. Seigers R, Schagen SB, Beerling W, et al. Long-lasting suppression of hippocampal cell proliferation and impaired cognitive performance by methotrexate in the rat. Behav Brain Res. 2008; 186: 168-175.

64. Madhyastha S, Somayaji SN, Rao MS, et al. Hippocampal brain amines in methotrexate-induced learning and memory deficit. Can J Physiol Pharmacol. 2002; 80: 1076-1084.

65. Igarashi $\mathrm{H}$, Inomata $\mathrm{K}$, \& Tateno A. The effect of methotrexate on the development of synapses in the neonatal rat hippocampus. Neuropediatrics. 1989; 20: 196-198.

66. Silverstein FS \& Johnston MV. A model of methotrexate encephalopathy: neurotransmitter and pathologic abnormalities. J Child Neurol. 1986; 1: 351-357.

67. Foley JJ, Raffa RB, \& Walker EA. Effects of chemotherapeutic agents 5-fluorouracil and methotrexate alone and combined in a mouse model of learning and memory. Psychopharmacology. 2008; 199: 527-538.

68. Winocur G, Vardy J, Binns MA, et al. The effects of anti-cancer drugs, methotrexate and 5-fluorouracil on cognitive function in mice. Pharmacol Biochem Behav. 2006; 85: 66-75.

69. Walker EA. Animal Models. In: Raffa RB and Tallarida RJ, ed. 'Chemo-fog': Cancer Chemotherapy-Related Cognitive Impairment. US: Landes Bioscience. 2010.

70. Chen D, Fu Wu C, Shi B, et al. Tamoxifen and toremifene cause impairment of learning and memory function in mice. Pharmacol Biochem Behav. 2002; 71: 269-276.

71. ELBeltagy M, Mustafa S, Umka J, et al. Fluoxetine improves the memory deficits caused by the chemotherapy agent 5-fluorouracil. Behav Brain Res. 2010; 208: 112-117.

72. Lyons L, ElBeltagy M, Umka J, et al. Fluoxetine reverses the memory impairment and reduction in proliferation and survival of hippocampal cells caused by methotrexate chemotherapy. Psychopharmacology. 2011; 215: 105-115.

73. van Praag H, Mei Qu P, Elliott RC, et al. Unilateral hippocampal lesions in newborn and adult rats: effects on spatial memory and BDNF gene expression. Behav Brain Res. 1998; 92: 21-30.

74. Mullenix PJ, Kernan WJ, Schunior A, et al. Interactions of steroid, methotrexate, and radiation determine neurotoxicity in an animal model to study therapy for childhood leukemia. Pediatr Res. 1994; 35: 171-178.

75. Sapolsky RM. A mechanism for glucocorticoid toxicity in the hippocampus: increased neuronal vulnerability to metabolic insults. J Neurosci. 1985; 5: 1228-1232.

76. Li CQ, Liu D, Huang L, et al. Cytosine arabinoside treatment impairs the remote spatial memory function and induces dendritic retraction in the anterior cingulate cortex of rats. Brain Res Bull. 2008; 77: 237-240.

77. Borzan J, LaGraize SC, \& Fuchs PN. Effect of chronic vincristine treatment on mechanical withdrawal response and pre-pulse inhibition in the rat. Neurosci Lett. 2004; 364: 110-113.

78. Eijkenboom M \& Van Der Staay FJ. Spatial learning deficits in rats after injection of vincristine into the dorsal hippocampus. Neuroscience. 1999; 91: 1299-1313.

79. Liedke PE, Reolon GK, Kilpp B, et al. Systemic administration of doxorubicin impairs aversively motivated memory in rats. Pharmacol Biochem Behav. 2009; 94: 239-243.

80. Sieklucka-Dziuba M, Saczonek J, Dziuba J, et al. Central action of some cytostatics-methotrexate (MTX) and doxorubicin (DXR): II. The influence on the seizure activity and the learning and memory processes in mice. Ann Univ Mariae Curie Sklodowska. 1998; 53: 81-88.
81. Macleod JE, DeLeo JA, Hickey WF, et al. Cancer chemotherapy impairs contextual but not cue-specific fear memory. Behav Brain Res. 2007; 181: 168-172.

82. Konat GW, Kraszpulski M, James I, et al. Cognitive dysfunction induced by chronic administration of common cancer chemotherapeutics in rats. Metab Brain Dis. 2008; 23: 325-333.

83. Heyser CJ. Assessment of developmental milestones in rodents. Curr Protoc Neurosci. 2003; 25: 8.18.1-8.18.15.

84. Laviola G, Macri S, Morley-Fletcher S, et al. Risk-taking behavior in adolescent mice: psychobiological determinants and early epigenetic influence. Neurosci Biobehav Rev. 2003; 27: 19-31.

85. Boyette-Davis JA \& Fuchs PN. Differential effects of paclitaxel treatment on cognitive functioning and mechanical sensitivity. Neurosci Lett. 2009; 453: 170-174.

86. Mondie CM, Vandergrift KA, Wilson $\mathrm{CL}$, et al. The chemotherapy agent, thioTEPA, yields long-term impairment of hippocampal cell proliferation and memory deficits but not depression-related behaviors in mice. Behav Brain Res. 2010; 209: 66-72.

87. Arnold HM, Bruno JP, \& Sarter M. Assessment of sustained and divided attention in rats. Curr Protoc Neurosci. 2003; 22: 8.5E.1-8.5E.13.

88. Seigers R, Pourtau L, Schagen SB, et al. Inhibition of hippocampal cell proliferation by methotrexate in rats is not potentiated by the presence of a tumor. Brain Res Bull. 2010; 81: 472-476.

89. Krull KR, Gioia G, Ness KK, et al. Reliability and validity of the childhood cancer survivor study neurocognitive questionnaire. Cancer. 2008; 113: 2188-97. 